\title{
Magnetic resonance imaging-guided lumbar nerve root infiltrations: optimization of an in-house protocol
}

\author{
Max Scheffler ${ }^{1 *}$ (D), Pauline Coralie Guillemin², Orane Lorton², Enrique Maturana', Nicolas Lauper ${ }^{3}$, \\ Dennis E. Dominguez ${ }^{3}$, Sylvain Terraz ${ }^{4}$, Pierre-Alexandre Poletti ${ }^{4}$, Rares Salomir ${ }^{2,4}$ and Sana Boudabbous ${ }^{4}$
}

\begin{abstract}
Background: For the treatment of radicular pain, nerve root infiltrations can be performed under MRI guidance in select, typically younger, patients where repeated CT exams are not desirable due to associated radiation risk, or potential allergic reactions to iodinated contrast medium.

Methods: Fifteen 3 T MRI-guided nerve root infiltrations were performed in 12 patients with a dedicated surface coil combined with the standard spine coil, using a breathhold PD sequence. The needle artifact on the MR images and the distance between the needle tip and the infiltrated nerve root were measured.

Results: The distance between the needle tip and the nerve root was $2.1 \pm 1.4 \mathrm{~mm}$. The visual artifact width, perpendicular to the needle long axis, was $2.1 \pm 0.7 \mathrm{~mm}$. No adverse events were reported.

Conclusion: This technical note describes the optimization of the procedure in a $3 \mathrm{~T}$ magnetic field, including reported procedure time and an assessment of targeting precision.
\end{abstract}

Keywords: MRI guidance, Nerve root infiltration, Radicular pain

\section{Background}

The burden of low back pain is substantial with an overall global prevalence estimated at $9 \%$, making it one of the leading causes of disability $[1,2]$. The etiology of low back pain is mostly degenerative and multifactorial. Degenerative changes not only trigger localized pain but may also give rise to radicular pain in the buttocks and lower limbs. It has been estimated that approximately $90 \%$ of sciatica, a common form of radicular pain, is due to disc herniation [3].

A number of interventional and surgical options are available for the treatment of radicular pain that is

\footnotetext{
*Correspondence: max.scheffler@hcuge.ch

1 Division of Radiology, Geneva University Hospitals, Ch. du Pont-Bochet

3, 1226 Thonex, Switzerland

Full list of author information is available at the end of the article
}

intractable to conservative medical management (including physical therapy), or is characterized by recurrence, with percutaneous infiltrations being the least invasive option. Infiltrations are typically performed under fluoroscopy, computed tomography (CT) or real-time CT guidance [4]. For CT-guided infiltrations a reported mean effective dose is $1.38 \mathrm{mSv}$ [5].

The age of onset of sciatica may occur earlier than low back pain, with a peak incidence thought to occur in patients in their forties [6]. Repeated CT- or fluoroscopyguided infiltrations, requested within an interval of a few months in younger patients, may be problematic from a radioprotection perspective. Furthermore, if the operator opts for an injection of iodinated contrast medium around the nerve, the patient is exposed to a risk of a contrast-induced allergic reaction, even if a very small amount is used. Magnetic resonance imaging (MRI) 
guidance in $1 \mathrm{~T}$ and $1.5 \mathrm{~T}$ magnetic fields has been used as a feasible and safe alternative [7-9]. MRI is free of ionizing radiation and offers a high soft tissue and fluid contrast but comes with the disadvantages of higher costs and a longer imaging time compared to CT [10].

We describe herein our MRI-guided infiltration method on a $3 \mathrm{~T}$ machine for the treatment of radicular pain. We quantitatively assessed the inclination of the MRI-conditional needle, the distance between the needle tip and the targeted nerve root, and the artifact created by the needle.

\section{Methods}

Fifteen MRI-guided nerve root infiltrations were carried out in 12 patients (mean age, 57 years \pm 18 SD; 6 females) on a 3 T MRI machine (Magnetom Skyra, Siemens Healthineers, Erlangen, Germany) between November 2017 and January 2021. Patients were consecutively selected by an expert board.

After confirmation of written consent, patients were placed on the MR table in a prone position. A dedicated surface coil proved to be necessary in addition to the standard spine coil. For this reason, we adapted a commercially available flexible surface coil (4-Channel Flex Large Coil, Siemens Healthineers, Erlangen, Germany) (Fig. 1a) in order to provide an enlarged entry window. The four central openings among the total of eight openings in the coil foam matrix were merged into two larger ones. The cut edges were resealed with epoxy resin and the openings reinforced by rigid polymer frames produced on a 3D printer, without modification of the radiofrequency resonant circuit. The widened openings offered sufficient manipulation space, even when the needle was significantly tilted. The coil was placed in the lumbar region, perpendicular to the long body axis, and one of the two large windows centered over the presumed point of skin puncture. The coil was attached with fastening straps to the MR table (Fig. 1b).

Standard T2-weighted turbo spin echo (TSE) sagittal and axial sequences were acquired for access planning. Main parameters of this sequences were: TE, $103 \mathrm{~ms}$; TR, $2300 \mathrm{~ms}$; flip angle, $150^{\circ}$; bandwidth, $260 \mathrm{~Hz} /$ pixel; acquisition matrix, $256 \times 140$; FOV, $300 \times 300 \mathrm{~mm}$; number of averages, 1; slice thickness, $2.5 \mathrm{~mm}$; acquisition time (AT), $14 \mathrm{~s}$ (one breathhold). Sagittal images confirmed the correct level to be infiltrated. A small silicone marker $(\varnothing=6 \mathrm{~mm}$, MR-Pinpoint 187, Beekley Medical, Bristol, USA) was placed on the chosen skin entry point and the coil position adjusted if necessary. In all cases we opted for a projected needle path where the nerve root could be reached as medially as possible, ideally inside in the neural foramen, in a strict axial plane.

The skin area under the coil's window was disinfected and covered with a perforated sterile film dressing. A

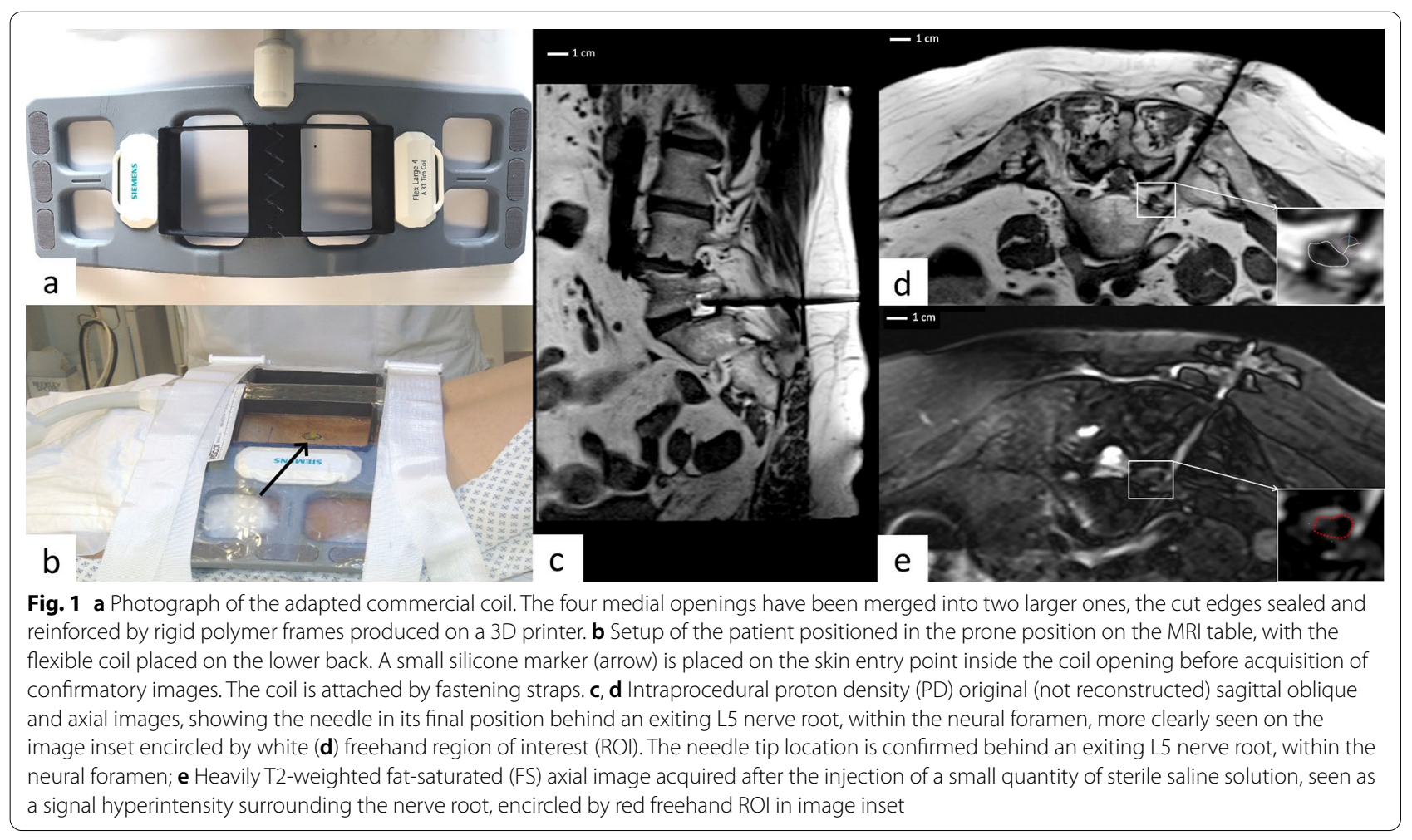


layer of sterile tissue drapes was used to cover the entire lower back area of the patient. Superficial and deep local anesthesia was performed with $1 \%$ lidocaine solution (Rapidocain 1\%, Sintetica, Mendrisio, Switzerland). Controlled by repeated proton density (PD)-weighted multi-slice axial and sagittal oblique breathhold acquisitions, an MRI-conditional 20G needle with a stylet (external diameter $0.9 \mathrm{~mm}$, Cytocut MRI, MDL, Delebio, Italy) was inserted and directed towards the exiting nerve root (Fig. 1c, d), aiming for an as short as possible needle-tip-nerve root distance. In some cases, a radiologic technologist facilitated adjustment of the needle's angulation with the assistance of a goniometer. The final position was determined intra-procedurally by the operator, taking into account possible radicular pain reported by the patient, always warranting a slight retreat of the needle. The PD sequence used for needle guidance had the following parameters: TE, $9.1 \mathrm{~ms}$; TR, $1030 \mathrm{~ms}$; flip angle, $132^{\circ}$; bandwidth, $199 \mathrm{~Hz} /$ pixel; acquisition matrix, $256 \times 252$; FOV, $240 \times 240 \mathrm{~mm}$; number of averages, 2 ; slice thickness, $2 \mathrm{~mm}$; acquisition plane, axial or sagittal oblique; acquisition time (AT), $17 \mathrm{~s}$ (one breathhold). To reduce imaging time, the slice stack coverage was limited to $11.6 \mathrm{~mm}$ (five $2 \mathrm{~mm}$-thick slices with a distance factor of $0.4 \mathrm{~mm}$ ). The selected phase encoding direction was anterior-posterior in order to minimize the chemical shift-like artifacts of the needle tip projecting along the readout direction.

With the needle tip positioned as close as possible behind the exiting nerve root and after a first attempt of aspiration, $1-2 \mathrm{ml}$ of sterile saline solution $(\mathrm{NaCl} 0.9 \%)$ were injected. A heavily T2-weighted fat-saturated single breathhold SPAIR (SPectral Attenuated Inversion Recovery) sequence was immediately performed to confirm the extravascular position of the needle tip and to visualize periradicular, and often epidural, diffusion of the fluid (Fig. 1e). This sequence had the following parameters: TE, $104 \mathrm{~ms}$; TR, $2420 \mathrm{~ms}$; flip angle, $150^{\circ}$; bandwidth, $260 \mathrm{~Hz} /$ pixel; acquisition matrix, $192 \times 154$; FOV, $128 \times 128 \mathrm{~mm}$; number of averages, 1 ; slice thickness, $3 \mathrm{~mm}$; AT, $18 \mathrm{~s}$ (one breathhold). If no fluid was visible on the SPAIR images, an intravascular position of the needle was thought to be likely and the needle moved back slightly, to inject another small amount of sterile saline.

After repeating the aspiration, injection of the active medication was performed. According to the preference of the requesting physician, we used $1 \mathrm{ml}$ of dexamethasone $0.4 \%$ solution (Mephameson $4 \mathrm{mg} / \mathrm{ml}$, Mepha Pharma, Basel, Switzerland) $\pm 1 \mathrm{ml}$ of ropivacaine $0.5 \%$ solution (Ropivacain $5 \mathrm{mg} / \mathrm{ml}$, Sintetica, Mendrisio, Switzerland).

Two metrics were used to assess the needle artifact on MR images. The first one consisted of a computer-based standardized measurement which did not correspond to the actual image guidance impairment but enabled a through-patient quantitative comparison. For this purpose, a 1D signal profile from the proton density axial image, cutting the needle perpendicularly, was approximated as an inversed Gaussian curve and the full width at half maximum (FWHM) was determined. The second metric used to evaluate the visual artifact, considered the subjective user-defined width of the dark band that artificially increased the needle geometric width.

The distance between the needle tip and the nerve root was calculated retrospectively on the most recent PD image prior to injection, aiming to demonstrate accurate targeting. The farthest left needle pixel, the farthest right needle pixel, and the lowermost needle pixel were used as starting points to draw individual lines to the posterior border of the nerve, with confirmation provided by the operators (M.S., S.B.) in doubtful cases. Procedure times were analyzed through measured room occupation times that included marking of the skin entry point and preparation of the sterile field.

\section{Results}

Results of the targeting end-point MRI data are shown in Table 1. The mean needle artifact according to the first metric was $3.8 \mathrm{~mm}$ (SD, $0.9 \mathrm{~mm}$ ). The mean needle artifact according to the second metric was $2.1 \mathrm{~mm}$ (SD, $0.7 \mathrm{~mm}$ ). The mean measured distance between the needle tip and the nerve root was $2.1 \mathrm{~mm}$ (SD, $1.4 \mathrm{~mm}$ ). The values were compared to the geometric diameter of the needle, $0.9 \mathrm{~mm}$, the ratios are provided in Table 1 . Periradicular \pm epidural diffusion of the injected normal saline could be observed in all patients before injection of the anti-inflammatory \pm anesthetic agents. Mean needle inclination was $19^{\circ}\left(\mathrm{SD}, 8^{\circ}\right)$. Mean MRI room occupation time was $51 \mathrm{~min}$. The quality of the images enabled increased confidence in technical success in all cases, independent of body habitus and despite the presence of severe spondylolisthesis in one patient. No patient experienced an adverse event during, or after, the procedure.

\section{Discussion}

In our series of fifteen $3 \mathrm{~T}$ MRI-guided interventions, good image quality and technical procedural success could be achieved in all cases, irrespective of individual body habitus. The adapted surface coil allowed for standard-of-care disinfection, facilitated access, and was resistant to cleaning with conventional disinfecting surface agents.

Despite some unavoidable susceptibility artifact from the needle, the distance between the tip and the exiting nerve root could be correctly determined. The transversal artifact of approximately $2 \mathrm{~mm}$ average 
Table 1 Quantitative results of 15 procedures in twelve patients, chronological order

\begin{tabular}{|c|c|c|c|c|c|c|c|c|}
\hline No & $\begin{array}{l}\text { Needle-nerve } \\
\text { root distance } \\
(\mathrm{mm})\end{array}$ & $\begin{array}{l}\text { Needle } \\
\text { angulation } \\
\left({ }^{\circ}\right)\end{array}$ & $\begin{array}{l}\text { MRI room } \\
\text { occupation time } \\
\text { (min) }\end{array}$ & $\begin{array}{l}\text { Nominal needle } \\
\text { diameter }(\mathrm{mm})\end{array}$ & $\begin{array}{l}\text { FWHM needle } \\
\text { artifact }(\mathrm{mm})\end{array}$ & $\begin{array}{l}\text { FWHM needle } \\
\text { artifact ratio* }\end{array}$ & $\begin{array}{l}\text { Visual } \\
\text { artifact } \\
(\mathrm{mm})\end{array}$ & $\begin{array}{l}\text { Visual } \\
\text { artifact } \\
\text { ratio }\end{array}$ \\
\hline 1 & 2.62 & 25.0 & 70 & 0.9 & 5.16 & 5.73 & 3.66 & 4.07 \\
\hline 2 & 3.56 & 27.9 & 55 & 0.9 & 4.94 & 5.49 & 3.06 & 3.40 \\
\hline 3 & 0.99 & 22.5 & 60 & 0.9 & 4.49 & 4.99 & 1.35 & 1.50 \\
\hline 4 & 1.95 & 22.7 & 65 & 0.9 & 3.98 & 4.42 & 2.68 & 2.98 \\
\hline 5 & 3.89 & 16.6 & 55 & 0.9 & 4.73 & 5.26 & 1.49 & 1.66 \\
\hline 6 & 2.69 & 13.0 & 54 & 0.9 & 4.73 & 5.26 & 2.34 & 2.60 \\
\hline 7 & 1.41 & 8.2 & 50 & 0.9 & 3.14 & 3.49 & 2.99 & 3.32 \\
\hline 8 & 4.73 & 21.2 & 64 & 0.9 & 2.57 & 2.86 & 1.54 & 1.71 \\
\hline 9 & 3.20 & 6.9 & 49 & 0.9 & 3.55 & 3.94 & 1.71 & 1.90 \\
\hline 10 & 0.10 & 24.6 & 40 & 0.9 & 4.84 & 5.38 & 1.83 & 2.03 \\
\hline 11 & 1.39 & 33.4 & 40 & 0.9 & 3.75 & 4.17 & 2.11 & 2.34 \\
\hline 12 & 1.89 & 14.3 & 39 & 0.9 & 3.04 & 3.38 & 2.12 & 2.36 \\
\hline 13 & 1.87 & 27.2 & 41 & 0.9 & 2.81 & 3.12 & 2.02 & 2.24 \\
\hline 14 & 0.10 & 12.4 & 44 & 0.9 & 3.05 & 3.39 & 1.49 & 1.66 \\
\hline 15 & 0.83 & 11.2 & 40 & 0.9 & 2.81 & 3.12 & 1.42 & 1.58 \\
\hline Mean & 2.08 & 19.1 & 51 & & 3.8 & 4.27 & 2.12 & 2.36 \\
\hline SD & 1.36 & 7.9 & 11.9 & & 0.91 & 1.01 & 0.69 & 0.78 \\
\hline
\end{tabular}

SD standard deviation, FWHM full width at half maximum

*Relative to physical diameter of the needle

width reported here for a 3 T MRI setup was significantly smaller than previously described in the literature at lower magnetic fields, for instance $6 \pm 0.2 \mathrm{~mm}$ for a $21 \mathrm{G}$ needle on a $1.5 \mathrm{~T}$ machine [7] and a range of $1.5-5 \mathrm{~mm}$ for a $20 \mathrm{G}$ needle on a $1 \mathrm{~T}$ machine [8]. The negligible longitudinal needle tip artifact was attributed to the fact that the phase encoding direction in the PD axial images avoided the chemical shiftlike artifact falsely prolonging the needle. Cases of our series showed distances between the needle tip and the nerve root of up to $4.7 \mathrm{~mm}$. The larger values could be explained by either clinical factors requiring a larger security margin independently of the intrinsic targeting accuracy (e.g. radicular pain signaled by the patient excluding further needle advancement), or an initially intravascular position of the needle tip that warranted a slight retreat. Some operators may prefer a $22 \mathrm{G}$ needle when performing a nerve root infiltration-while being less traumatic it may also be more prone to flexion and deviation from a chosen access path-and smaller MR artifacts should be expected in that case, although this also depends on the manufacturing alloy.

The room occupancy time decreased over the period of the 15 procedures, with 40 min needed for the last intervention, in comparison to $70 \mathrm{~min}$ for the first. As $3 \mathrm{~T}$ MRI enables the performance of very rapid PD sequences (within one single breathhold), the main axis of improvement lies in the patient's set-up time and the orchestration of single steps of the infiltration procedure itself. With select patients we can expect to reach an MRI room occupancy time between 25 and $35 \mathrm{~min}$.

Contrarily to CT, MRI scanners do not have an inclinable gantry but offer freely definable acquisition planes. In our series however, all procedures could be realized in a strict axial plane, with a mean needle angulation of $19.1^{\circ}$.

\section{Conclusion}

In conclusion, for a select group of patients suffering from radicular pain, lumbar nerve root infiltrations could be performed radiation-free and without iodinated contrast medium using 3 T MRI. Our method offers an optimized procedural workflow with high image quality which can be performed within reasonable times and without compromising safety.

\section{Abbreviations}

CT: Computed tomography; MRI: Magnetic resonance imaging; PD: Proton density; SD: Standard derivation; FWHM: Full width at half maximum; FOV: Field of view; AT: Acquisition time; TR: Repetition time; TE: Echo time; TSE:

Turbo spin echo; SPAIR: SPectral Attenuated Inversion Recovery; ROI: Region of interest.

\section{Acknowledgements}

Not applicable. 


\section{Authors' contributions}

MS, PCG and RS performed the main writing of the article. PCG collected and organized data in spreadsheet form, performed retrospective measures. PCG, OL and RS technically adapted the used MR coil. PCG and RS prepared the article's images. EM and RS were responsible for the main contributions in improving and optimizing the MR acquisition sequences. Critiques and corrections were provided by OL, NL, DD, ST, and PAP. RS and SB designed the work and oversaw its progress and final presentation giving continuous feedback. All authors read and approved the final manuscript.

\section{Funding}

The study was supported by an institutional grant from the Department of Radiology and Medical Informatics, Faculty of Medicine, University of Geneva, Geneva, Switzerland.

\section{Availability of data and materials}

The images of Fig. 1 can be obtained as DICOM files from the corresponding author.

\section{Declarations}

\section{Ethics approval and consent to participate}

All procedures performed in the studies involving human participants were in accordance with the ethical standards of the institutional and/or national research committee (CCER, Geneva, Switzerland, granted approval decision no. 2019-02301) and with the 1964 Helsinki Declaration and its later amendments or comparable ethical standards. Informed written consent was obtained from all individual participants included in the study.

\section{Consent for publication}

Not applicable.

\section{Competing interest}

The authors declare that they have no conflict of interest.

\section{Author details}

'Division of Radiology, Geneva University Hospitals, Ch. du Pont-Bochet 3, 1226 Thonex, Switzerland. ${ }^{2}$ Division of Radiology, University of Geneva, Rue Gabrielle-Perret-Gentil 4, 1205 Geneva, Switzerland. ${ }^{3}$ Division of Orthopedic Surgery and Traumatology, Geneva University Hospitals, Rue Gabrielle-Perret-Gentil 4, 1205 Geneva, Switzerland. ${ }^{4}$ Division of Radiology, Geneva University Hospitals, Rue Gabrielle-Perret-Gentil 4, 1205 Geneva, Switzerland.
Received: 14 February 2021 Accepted: 6 July 2021

Published online: 12 July 2021

\section{References}

1. Smith E, Hoy DG, Cross M, et al. The global burden of other musculoskeletal disorders: estimates from the Global Burden of Disease 2010 study. Ann Rheum Dis. 2014;73:1462-9.

2. Vos T, Flaxman AD, Naghavi M, et al. Years lived with disability (YLDs) for 1160 sequelae of 289 diseases and injuries 1990-2010: a systematic analysis for the Global Burden of Disease Study. Lancet. 2010;2012:2163-96.

3. Valat JP, Genevay S, Marty M, et al. Sciatica. Best Pract Res Clin Rheumatol. 2010:241-252.

4. Lewis RA, Williams NH, Sutton AJ, et al. Comparative clinical effectiveness of management strategies for sciatica: systematic review and network meta-analyses. Spine J. 2015:1461-77.

5. Artner J, Lattig F, Reichel H, Cakir B. Effective dose of CT-guided epidural and periradicular injections of the lumbar spine: a retrospective study. Open Orthop J. 2012:357-361.

6. Davis D, Maini K, Vasudevan A (2020) Sciatica. StatPearls Publishing. https://www.ncbi.nlm.nih.gov/books/NBK507908. Accessed 12 November 2020.

7. Fritz J, Thomas C, Clasen S, et al. Freehand real-time MRI-guided lumbar spinal injection procedures at 1.5 T: feasibility, accuracy, and safety. AJR. 2009:W161-7.

8. Streitparth F, De Bucourt M, Hartwig T, et al. Real-time MR-guided lumbosacral periradicular injection therapy using an open 1.0-T MRI system: an outcome study. Invest Radiol. 2013:471-6.

9. Streitparth $F$, Walter T, Wonneberger $U$, et al. Image-guided spinal injection procedures in open high-field MRI with vertical field orientation: Feasibility and technical features. Eur Radiol. 2010:395-403.

10. Maurer MH, Schreiter N, De Bucourt M, et al. Cost comparison of nerve root infiltration of the lumbar spine under MRI and CT guidance. Eur Radiol. 2013:1487-94.

\section{Publisher's Note}

Springer Nature remains neutral with regard to jurisdictional claims in published maps and institutional affiliations.

Ready to submit your research? Choose BMC and benefit from:

- fast, convenient online submission

- thorough peer review by experienced researchers in your field

- rapid publication on acceptance

- support for research data, including large and complex data types

- gold Open Access which fosters wider collaboration and increased citations

- maximum visibility for your research: over $100 \mathrm{M}$ website views per year

At BMC, research is always in progress.

Learn more biomedcentral.com/submissions 